\title{
A Nomogram to Assess Small-Intestinal Neuroendocrine Tumor ('Carcinoid') Survival
}

\author{
Irvin M. Modlin ${ }^{a} \quad$ Bjorn I. Gustafsson ${ }^{a, b} \quad$ Marianne Pavel ${ }^{c} \quad$ Bernhard Svejda ${ }^{a}$ \\ Benjamin Lawrence $^{\mathrm{a}}$ Mark Kidd $^{\mathrm{a}}$ \\ aGastrointestinal Pathobiology Research Group, Yale University School of Medicine, New Haven, Conn., USA; \\ ${ }^{b}$ Department of Cancer Research and Molecular Medicine, Norwegian University of Science and Technology, \\ Trondheim, Norway; ' Medizinische Klinik mit Schwerpunkt Hepatologie und Gastroenterologie, Charité, \\ Campus-Virchow-Klinikum, Universitätsmedizin Berlin, Berlin, Germany
}

\section{Key Words}

Carcinoid $\cdot$ Cox model $\cdot$ Hazard ratio $\cdot$ Kaplan-Meier method $\cdot$ Modlin Score $\cdot$ National Cancer Institute • Neuroendocrine tumor nomogram - Surveillance, Epidemiology and End Results database - Small intestine • Survival prediction

\begin{abstract}
Neuroendocrine tumors (NETs) are a heterogeneous group of cancers of which the commonest site is the small intestine (SI). Most information available to determine tumor behavior reflects univariate assessment of factors or is anecdotal or experience based. There currently exists no objective multivariate analysis of indices that defines SI NET prognosis. A key unmet need is the lack of a rigorous mathematical-based tool - a nomogram - for the assessment of parameters that define progress, determine prognosis and can guide therapy. Since prediction of NET behavior is a critical criterion in determining clinical strategy, we constructed a NET nomogram (Modlin Score) for prognosis prediction, patient group comparisons and a guide for stratification of treatment and surveillance. We used hazard ratio (HR), Cox analysis and Kaplan-Meier analysis of published data and the current Sur-
\end{abstract}

veillance, Epidemiology and End Results (SEER) database (approx. 20,000 patients) to develop a nomogram from 15 variables demonstrated to provide significant multivariate HRs. These included age, gender, ethnicity, symptoms, urinary 5-hydroxyindoleacetic acid, plasma chromogranin A, liver function tests, tumor size, invasion, metastasis, histology, Ki-67 index, carcinoid heart disease and therapy (surgery or long-acting somatostatin analogs). Internal validation was assessed using 33 SI NET patients. A NET nomoscore (Modlin Score) was developed by HR weighting and stratification into low $(<75)$, medium (75-95) and high risk ( $>95)$. This identified significant differences $(p<0.03$, Kaplan-Meier) in survival $(15.5 \pm 4.3,9.7 \pm 2.5$ and $6.4 \pm 1.1$ years, respectively). The Modlin Score was significantly elevated ( $p<$ $0.01)$ in deceased compared to alive patients. This nomogram represents an optimized construct based upon currently analyzable data, and application will facilitate accurate stratification for comparison in clinical trials. External validation and amplification by identification of additional indices, e.g. molecular biomarkers, are necessary. The development of a mathematically validated nomogram provides a platform for objective assessment of SI NET disease, a finite basis for precise prognostication and a tool to guide management strategy.

\section{KARGER}

Fax +4161306 1234

E-Mail karger@karger.ch

www.karger.com
C) 2010 S. Karger AG, Basel

$0028-3835 / 10 / 0923-0143 \$ 26.00 / 0$

Accessible online at:

www.karger.com/nen
Irvin M. Modlin

Department of Gastroenterological Surgery, Yale University School of Medicine 333 Cedar Street, PO Box 208062

New Haven, CN 06520-8062 (USA)

Tel. +1 203785 5429, Fax +1 203737 4067, E-Mail imodlin@ optonline.net 


\section{Introduction}

Neuroendocrine tumors (NETs) represent both a relatively rare neoplastic process as well as a diverse group of tumors segregated within a group previously considered as 'carcinoids' $[1,2]$. The tumors occur in numerous sites but are especially common within the gastrointestinal tract and the bronchopulmonary system reflecting the wide distribution of their neuroendocrine cells of origin (e.g. enterochromaffin cells, gastrin cells, $\beta$-cells) which are sensory and regulatory in function $[2,3]$. Relatively little is known of the etiology of the disease, and each NET, depending on its anatomical site, arises from a different neuroendocrine cell and exhibits a different functionality as well as significantly different biological and malignant behavior $[2,4]$. Thus, prediction of prognosis and outcome of an individual NET is difficult and, in many instances, inaccurate. This reflects the absence of any organized assessment system to provide a mathematical basis for objective appraisal of disease stratification and risk analysis. The development of a nomogram that can be utilized to provide a prognostic model to predict disease-specific death for patients with a NET remains a major unmet need in the discipline of neuroendocrine oncology.

Patients with a NET have an unpredictable survival even when there is successful resection of the primary tumor and/or its metastases, which is due to the biological heterogeneity of the tumors. Since these cancers have differing genetic, cellular and behavioral characteristics, their survival is not uniform. Patient prognosis is currently estimated on the basis of a number of different systems proposed by the American Joint Committee on Cancer (AJCC), World Health Organization (WHO) and European Neuroendocrine Society (ENETS) [5-9]. These are variably based upon prognostic determinants such as histological differentiation (well-differentiated NET/ neuroendocrine carcinoma and poorly differentiated neuroendocrine carcinoma and biological/pathomorphological signs of malignancy, or more recently on the TNM staging system) $[2,5-7,9,10]$. By integrating additional significant prognostic factors, a nomogram can be developed to not only better assess an individual patient's disease-specific survival, but also provide information that may be helpful in defining treatment options or comparing treatment groups.

In the USA, the incidence (2003-2007) of the disease based upon the 2007 National Cancer Institute's (NCI) Surveillance, Epidemiology and End Results (SEER) database was 5.76/100,000, and the prevalence in 2004 was estimated to be approximately $35 / 100,000[11,12]$. The incidence is equivalent to esophageal cancer $(4.5 / 100,000)$, testicularcancer $(5.4 / 100,000)$ and myeloma $(5.4 / 100,000)$. The prevalence renders gastroenteropancreatic NETs (GEP NETs) the second most common gastrointestinal cancer after colon cancer, and more prevalent than pancreatic, gastric, esophageal or hepatic cancer or any two of these combined [13].

Approximately 18,000 cases and 8,200 deaths attributable to this disease are predicted for 2011 in the USA based on the NCI SEER data [11]. Given the wide range of the 5 -year survival rate of $41-87 \%$ depending on disease extent, grade and tumor site [11], patients with a NET require an accurate prognosis. With accurate prediction, patients at low risk for disease-specific death can be safely reassured, whereas patients at high risk can be considered for appropriate surgery and systemic therapy [2]. Several studies have identified prognostic factors in individual NETs but a rigorous and robust assimilation of the different indices used to define outcome is lacking [12, 14-23]. Although assessment of a variety of parameters and knowledge thereof has utility in clinicopathological research and clinical trial design, patient-specific counseling and therapeutic strategy require formalized integration of diverse prognostic factors to establish a single patient-specific prognosis. In addition, the ability of such a tool, a nomogram, to generate individualized predictions, will facilitate the identification and stratification of patients in clinical trials [24].

It is evident that a simplistic enumeration of the risk factors of an individual is insufficient to objectively integrate or adequately weight the information available for prediction. A standardized inventory of risk factors erroneously assumes that each factor has equal or equivalent weight, and if a continuous variable, such as patient age at diagnosis, is categorized for counting, the information quotient may be diminished [25]. In order to best construct a nomogram, the range of variables ideally considered should be determined based on data availability and clinical evidence as well as on statistical significance (using modeling programs, e.g. Cox proportional hazards model). Prognostic parameters therefore need to be identified based on both research and clinical rationale, and then weighted according to the size of the prognostic effect ideally allowing for the differing variance in each sample. As such, multivariate hazard ratios (HRs) can be readily compared across separate studies and can translate directly into weightings for each prognostic variable.

The purpose of this study was to develop a prognostic model, or nomogram, that predicts disease-specific death 
for patients with NET disease of the small intestine (SI; 'carcinoid'). We used information acquired from current literature resources (12,412 patients) and 7,445 SI NET patients identified within the 1977-2007 NCI SEER database to create multivariate HRs for each prognostic factor. Multiple statistical modeling strategies were compared for their ability to combine the established prognostic factors into a model that predicts accurately, and this final model was internally validated.

\section{Materials and Methods}

Detection of variables that predict survival required HRs which were obtained from the literature or calculated de novo from the latest iteration of the NCI SEER database (2007).

\section{Literature Assessment to Identify Appropriate Prognostic}

Indices

A retrospective survey of the current status of risk (HRs) associated with gastrointestinal NETs (and specifically SI 'carcinoids' and NETs) was undertaken using the PubMed database (available at URL: http://www.pubmed.gov, accessed June 6, 2010). Key words used in the literature search included carcinoid, gastrointestinal, hazard ratio (HR), Kaplan-Meier, multivariate analysis, neuroendocrine tumor, small intestine and survival (table 1). Research articles were evaluated for survival information, particularly with reference to univariate (table 2) and multivariate (table 3) HRs. All relevant articles ( $\mathrm{n}=19$ articles, 12,412 patients) were published from 1997 to 2010.

\section{Analysis of SEER Database (1977-2007) for Indices}

The patient population is derived from the NCI SEER database. A search of the database identified 24,850 patients with gastrointestinal NETs (stomach, small bowel, colon, rectum and appendix) between 1977 and 2007. Of these patients, 7,445 had SI NETs (table 4). Patients with a diagnosis of Meckel's diverticulum carcinoids $(n=133)$ were included. Patients with other causes of death were excluded. Clinicopathological factors were analyzed to determine the effect on overall survival using the log rank test (Mantel-Cox). Multivariate analysis was performed using the Cox proportional hazards model (SPSS 16.0, IBM). Variables were added to the multivariate model based on a stepwise forward (Wald statistic model) selection procedure where the entry criterion for each variable was based on $p<0.05$. Variables evaluated included gender, ethnicity (race: White, Black and other - including Hispanic, Latino and unknown), SEER stage or extent (local, regional or distant disease) and degree of differentiation/grade. The latter included well-differentiated (grade I), moderately differentiated (grade II), poorly differentiated (grade III), undifferentiated (anaplastic: grade IV) and unknown. Different classification systems are used in the USA and Europe so a combination of both the WHO and TNM were examined. Survival curves were generated from Kaplan-Meier analysis (fig. 1), and statistically significant variables were included in table 4 and used to generate the nomogram (fig. 2).

Nomogram to Assess Small-Intestinal

Neuroendocrine Tumor Survival
Table 1. Potentially assessable prognostic indices

Factor

Data

available

Patient characteristics

Age yes

Gender yes

Ethnicity/race ${ }^{1} \quad$ yes

Symptoms

Bioactive amine/peptide-related symptoms ${ }^{2} \quad$ yes

Biochemistry, urine

5-HIAA levels ${ }^{3}$

Biochemistry (blood)

Elevated CgA blood levels ${ }^{4} \quad$ yes

Liver function tests

Abnormal liver function tests ${ }^{5}$ yes

Tumor morphology, extent and immunohistochemistry Histological grade yes

CgA staining ('well differentiated') yes

Tumor size yes

Ki-67 index yes

Mitoses $^{6}$ no

SSTR2 expression no

Disease topography

Local disease only yes

Regional disease

(involving regional lymph nodes) yes

Metastasis (liver/elsewhere) yes

Presence of carcinoid heart disease ${ }^{7} \quad$ yes

Therapeutic intervention

Surgery - with curative intent yes

Surgery - primary resectable/residual disease yes

Tumor debulking (hepatic/mesenteric) yes

Response to systemic therapy yes

Performance status

Karnofsky score yes

5-HIAA = 5-Hydroxyindoleacetic acid; $\mathrm{CgA}=$ chromogranin A; SSTR = somatostatin receptor.

${ }^{1}$ Ethnicity/race as defined by NCI SEER database (White, Black, other). ${ }^{2}$ Symptoms of 'carcinoid syndrome'. ${ }^{3}$ Elevation above normal range. ${ }^{4}>6$ times the upper limit of normal. ${ }^{5}$ Elevation greater than the upper normal limit of bilirubin, alkaline phosphatase or $\gamma$-glutamyltransferase. ${ }^{6}$ Mitoses per 10 high-powered field on histological examination. ${ }^{7}$ Echocardiogram.

\section{Development of the NET Nomogram}

Statistically significant variables identified either in the literature review or from the multivariate analysis of the most recent NCI SEER database are shown in table 5. Patient age, gender, ethnicity, presence of specific symptoms at diagnosis (flushing, diarrhea), elevated urinary 5-hydroxyindoleacetic acid (5-HIAA; $>300$ $\mu \mathrm{mol} / 24 \mathrm{~h}$ ), elevated plasma chromogranin A ( $>6 \times$ upper limit of normal), abnormal liver function studies, tumor size, SEER staging, histology (grade), Ki-67 index, the presence of carcinoid heart disease, liver metastases (detected by any conventional modality, 
Table 2. Literature compilation of univariate variables predictive of survival in SI NETs

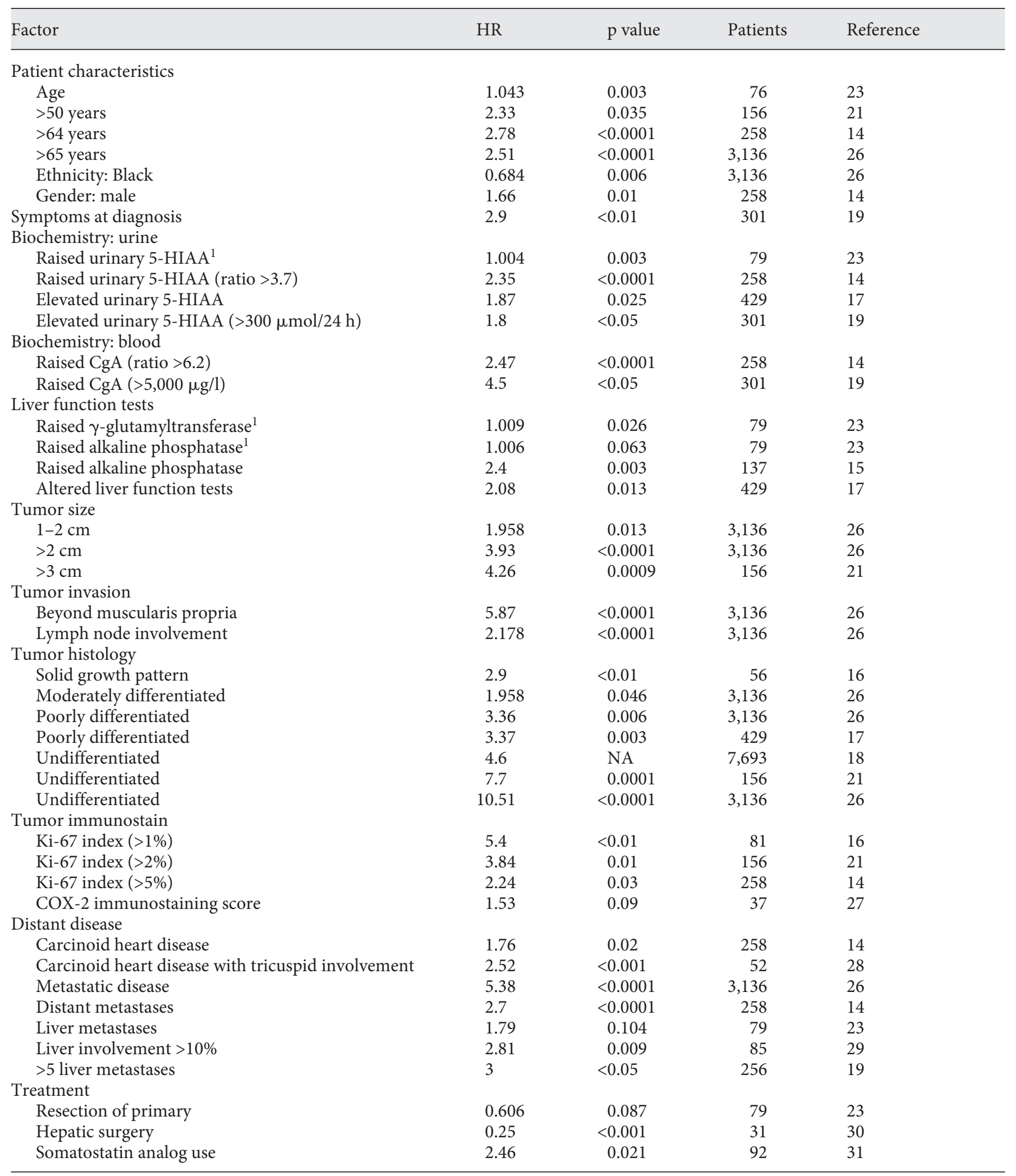

5-HIAA = 5-Hydroxyindoleacetic acid; CgA = chromogranin A; COX = cyclooxygenase ${ }^{1} \mathrm{HR}$ per year. 
Table 3. Compilation of multivariate variables predictive of survival in SI NETs from the literature and current (1973-2007) SEER analysis

\begin{tabular}{|c|c|c|c|c|}
\hline Factor & HR & $\mathrm{p}$ value & Patients & Reference \\
\hline \multicolumn{5}{|l|}{ Patient characteristics } \\
\hline Age, per year & 1.052 & 0.001 & 76 & 23 \\
\hline Age, per year & 1.02 & 0.006 & 200 & 32 \\
\hline $55-74$ years & 1.9 & $<0.0001$ & 3,231 & 20 \\
\hline $55-74$ years & 2.26 & NA & 7,693 & 18 \\
\hline$>62$ years & 3.4 & 0.0001 & 154 & 33 \\
\hline$>64$ years & 3.12 & $<0.001$ & 258 & 14 \\
\hline$>65$ years & 3.372 & $<0.0001$ & 3,136 & 26 \\
\hline$>65$ years & 1.91 & $<0.001$ & 3,175 & 20 \\
\hline$>75$ years & 3.58 & NA & 7,693 & 18 \\
\hline Gender: female & 0.8 & $<0.0001$ & 3,231 & 20 \\
\hline Ethnicity & 1.1 & 0.006 & 7,445 & SEER 2007 \\
\hline Symptoms at diagnosis & 8.2 & 0.04 & 399 & 34 \\
\hline \multicolumn{5}{|l|}{ Biochemistry: urine } \\
\hline Raised urinary 5-HIAA & 1.003 & 0.03 & 79 & 3 \\
\hline Elevated urinary 5-HIAA & 2.36 & 0.006 & 429 & 17 \\
\hline Elevated urinary 5-HIAA & 1.11 & 0.02 & 200 & 32 \\
\hline \multicolumn{5}{|l|}{ Biochemistry: blood } \\
\hline Raised CgA (ratio >6.2) & 1.90 & 0.02 & 258 & 14 \\
\hline Raised CgA $(>5,000 \mu \mathrm{g} / \mathrm{l})$ & 4.4 & $<0.01$ & 301 & 19 \\
\hline \multicolumn{5}{|l|}{ Liver function tests } \\
\hline Raised $\gamma$-glutamyltransferase & 1.009 & 0.002 & 79 & 23 \\
\hline Altered liver function tests & 2.21 & 0.02 & 429 & 17 \\
\hline \multicolumn{5}{|l|}{ Tumor size } \\
\hline Primary tumor $>2 \mathrm{~cm}$ & 2.83 & $<0.0001$ & 3,136 & 26 \\
\hline Primary tumor $>2.5 \mathrm{~cm}$ & 4.44 & $<0.001$ & 399 & 34 \\
\hline \multicolumn{5}{|l|}{ Tumor invasion } \\
\hline Increasing SEER staging & 1.21 & $<0.0001$ & 7,445 & SEER 2007 \\
\hline Beyond muscularis propria & 2.97 & 0.009 & 3,136 & 26 \\
\hline \multicolumn{5}{|l|}{ Tumor histology } \\
\hline Increasing histological grade & 1.07 & $<0.0001$ & 7,445 & SEER 2007 \\
\hline Poorly differentiated & 2.99 & 0.034 & 156 & 21 \\
\hline Poorly differentiated & 4.02 & 0.02 & 429 & 17 \\
\hline \multicolumn{5}{|l|}{ Tumor immunohistochemistry } \\
\hline Ki-67 index $(>5 \%)$ & 3.99 & 0.01 & 399 & 34 \\
\hline Ki-67 index $(>10 \%)$ & 24.8 & $<0.001$ & 399 & 34 \\
\hline \multicolumn{5}{|l|}{ Distant disease } \\
\hline Carcinoid heart disease & 2.04 & 0.001 & 200 & 32 \\
\hline Carcinoid heart disease with tricuspid involvement & 2.55 & $<0.001$ & 52 & 28 \\
\hline Distant metastases & 1.98 & 0.04 & 258 & 14 \\
\hline Liver metastases & 2.3 & 0.003 & 95 & 33 \\
\hline Liver involvement $>10 \%$ & 2.63 & 0.002 & 85 & 29 \\
\hline \multicolumn{5}{|l|}{ Treatment } \\
\hline Resection of primary & 0.581 & 0.097 & 79 & 23 \\
\hline Surgery & 0.21 & $<0.001$ & 399 & 34 \\
\hline Hepatic surgery & 0.31 & 0.003 & 31 & 30 \\
\hline Somatostatin analog use & 0.27 & $<0.001$ & 85 & 29 \\
\hline
\end{tabular}

5-HIAA = 5-Hydroxyindoleacetic acid; $\mathrm{CgA}=$ chromogranin $\mathrm{A} ; \mathrm{NA}=$ not available . 
Table 4. Clinicopathological characteristics and univariate analysis of SI NETs from the current SEER database (1973-2007): patient demographics and survival $(\mathrm{n}=7,445)$

\begin{tabular}{lrrlll}
\hline $\begin{array}{l}\text { Indices/ } \\
\text { detail }\end{array}$ & Number & $\begin{array}{l}\text { Mean } \\
\text { survival } \\
\text { months }\end{array}$ & $\begin{array}{l}\text { Standard } \\
\text { error }\end{array}$ & & $\begin{array}{l}\mathrm{p} \\
\text { value }\end{array}$ \\
\hline Gender & & & & & \\
$\quad$ Female & 3,519 & 126.1 & 3.1 & 1.27 & 0.26 \\
$\quad$ Male & 3,926 & 130.5 & 3.1 & & \\
Ethnicity & & & & & \\
White & 6,449 & 129 & 2.45 & & \\
Black & 833 & 118 & 6.2 & 8.58 & $<0.014$ \\
Other & 163 & 94 & 8 & & \\
Grade & & & & & \\
WD, I & 690 & 149.5 & 12.9 & & \\
MD, II & 243 & 115.3 & 12.8 & & \\
PD, III & 85 & 77.6 & 11.9 & 53.46 & $<0.001$ \\
UD/A, IV & 29 & 51.9 & 10.8 & & \\
$\quad$ Unknown & 6,398 & 127.1 & 2.4 & & \\
Extent & & & & & \\
Localized & 2,039 & 145.1 & 5.3 & & \\
Regional & 3,015 & 145.4 & 3.6 & 204.38 & $<0.0001$ \\
Distant & 2,164 & 90.6 & 2.8 & & \\
Unknown & 227 & 104.6 & 9.2 & & \\
\hline
\end{tabular}

$\chi^{2}$ : Reflects the log rank (Mantel-Cox) $\chi^{2}$ score from KaplanMeier analysis. Grades: WD, I = well-differentiated grade I; MD, $\mathrm{II}$ = moderately differentiated grade II; PD, III = poorly differentiated grade III; UD/A, IV = undifferentiated (anaplastic) grade IV; unknown $=$ not graded in the NCI SEER database .

e.g. CT or MRI or combination), and whether patients had undergone hepatic surgery or somatostatin analog therapy were identified to be the most statistically significant prognostic factors relating to survival. An SI NET prognostic 'survival' score for each variable was then developed by direct translation of the regression coefficients or HR (table 5). The points were multiplied by 4 to generate a $0-100$ scale and then summed into a raw score.

Internal validation was undertaken using patients from Yale University School of Medicine, New Haven, Conn., USA ( $n=8)$, St. Olav's Hospital, Trondheim, Norway $(n=9)$, and the Klinik für Hepatologie und Gastroenterologie, Charité, Campus-VirchowKlinikum, Berlin, Germany $(\mathrm{n}=16)$. The demographics of this group was: median age 63 years (range $42-80$ ), the M:F ratio 20:13, and ethnicity 32 White, 1 Black, 0 other (Asian). The observed follow-up was $0.5-19$ years, and overall survival was $9.1 \pm$ 1.3 years. Nomogram scores were compared between alive and deceased patients (Mann-Whitney 2-tailed test) while the predictive utility of the nomogram scores was assessed using KaplanMeier survival analysis [14, 34] (fig. 3).
Table 5. Prognostic score criteria and point allocation for NET nomogram development

\begin{tabular}{|c|c|c|c|}
\hline Indices & & $\begin{array}{l}\text { Points } \\
\text { estimated } \\
\text { from HR }\end{array}$ & $\begin{array}{l}\text { Calculated } \\
\text { nomoscore } \\
\text { points }\end{array}$ \\
\hline Age $>62$ years & & 3 & 12 \\
\hline \multirow[t]{2}{*}{ Gender } & Female & 0 & 0 \\
\hline & Male & 1 & 4 \\
\hline \multirow[t]{3}{*}{ Ethnicity $^{1}$} & White & 0 & 0 \\
\hline & Black & 1 & 4 \\
\hline & Other (Hispanic) & 2 & 8 \\
\hline \multirow{2}{*}{$\begin{array}{l}\text { Symptoms at } \\
\text { diagnosis }^{2}\end{array}$} & None & 0 & 0 \\
\hline & Yes & 8 & 32 \\
\hline \multirow{2}{*}{$\begin{array}{l}\text { Elevated 5-HIAA } \\
(>2 \times \text { ULN })\end{array}$} & No & 0 & 0 \\
\hline & Yes & 1 & 4 \\
\hline \multirow{2}{*}{$\begin{array}{l}\text { Elevated CgA } \\
(>6 \times \text { ULN) }\end{array}$} & No & 0 & 0 \\
\hline & Yes & 2 & 8 \\
\hline \multirow{2}{*}{$\begin{array}{l}\text { Abnormal liver } \\
\text { function tests }{ }^{3}\end{array}$} & No & 0 & 0 \\
\hline & Yes & 2 & 8 \\
\hline \multirow[t]{3}{*}{ Tumor size } & $<2 \mathrm{~cm}$ & 0 & 0 \\
\hline & $2-2.5 \mathrm{~cm}$ & 2 & 8 \\
\hline & $>2.5 \mathrm{~cm}$ & 3 & 12 \\
\hline \multirow{3}{*}{$\begin{array}{l}\text { Tumor invasion } \\
\text { (SEER stage) }\end{array}$} & Localized & 0 & 0 \\
\hline & Regional & 1 & 4 \\
\hline & Distant & 3 & 12 \\
\hline \multirow[t]{4}{*}{ Tumor histology } & Grade I & 1 & 4 \\
\hline & Grade II & 2 & 8 \\
\hline & Grade III & 3 & 12 \\
\hline & Grade IV & 4 & 16 \\
\hline \multirow[t]{3}{*}{ Ki-67 index } & $<5$ & 0 & 0 \\
\hline & $5-10$ & 4 & 16 \\
\hline & $>10$ & 25 & 100 \\
\hline \multirow{2}{*}{$\begin{array}{l}\text { Carcinoid } \\
\text { heart disease }^{4}\end{array}$} & No & 0 & 0 \\
\hline & Yes & 2 & 8 \\
\hline \multirow[t]{2}{*}{ Liver metastases } & No & 0 & 0 \\
\hline & Yes & 2 & 8 \\
\hline \multirow[t]{2}{*}{ Surgical therapy 5} & None & 2 & 8 \\
\hline & Yes & 0 & 0 \\
\hline \multirow[t]{2}{*}{ Medical therapy } & None & 3 & 12 \\
\hline & SST analog & 0 & 0 \\
\hline
\end{tabular}

A linear score for age was developed based on an HR of 1.05/year (from table 3). 5-HIAA = 5-Hydroxyindoleacetic acid; $\mathrm{CgA}=$ chromogranin A; ULN = upper limit of normal.

${ }^{1}$ Ethnicity/race as defined by NCI SEER database (White, Black, other/Hispanic).

${ }^{2}$ Symptoms - 'carcinoid syndrome'.

${ }^{3}$ Elevation beyond upper limit for bilirubin, alkaline phosphatase or $\gamma$-glutamyltransferase.

${ }^{4}$ Confirmed by echocardiography.

${ }^{5}$ Surgical resection of hepatic metastases. 


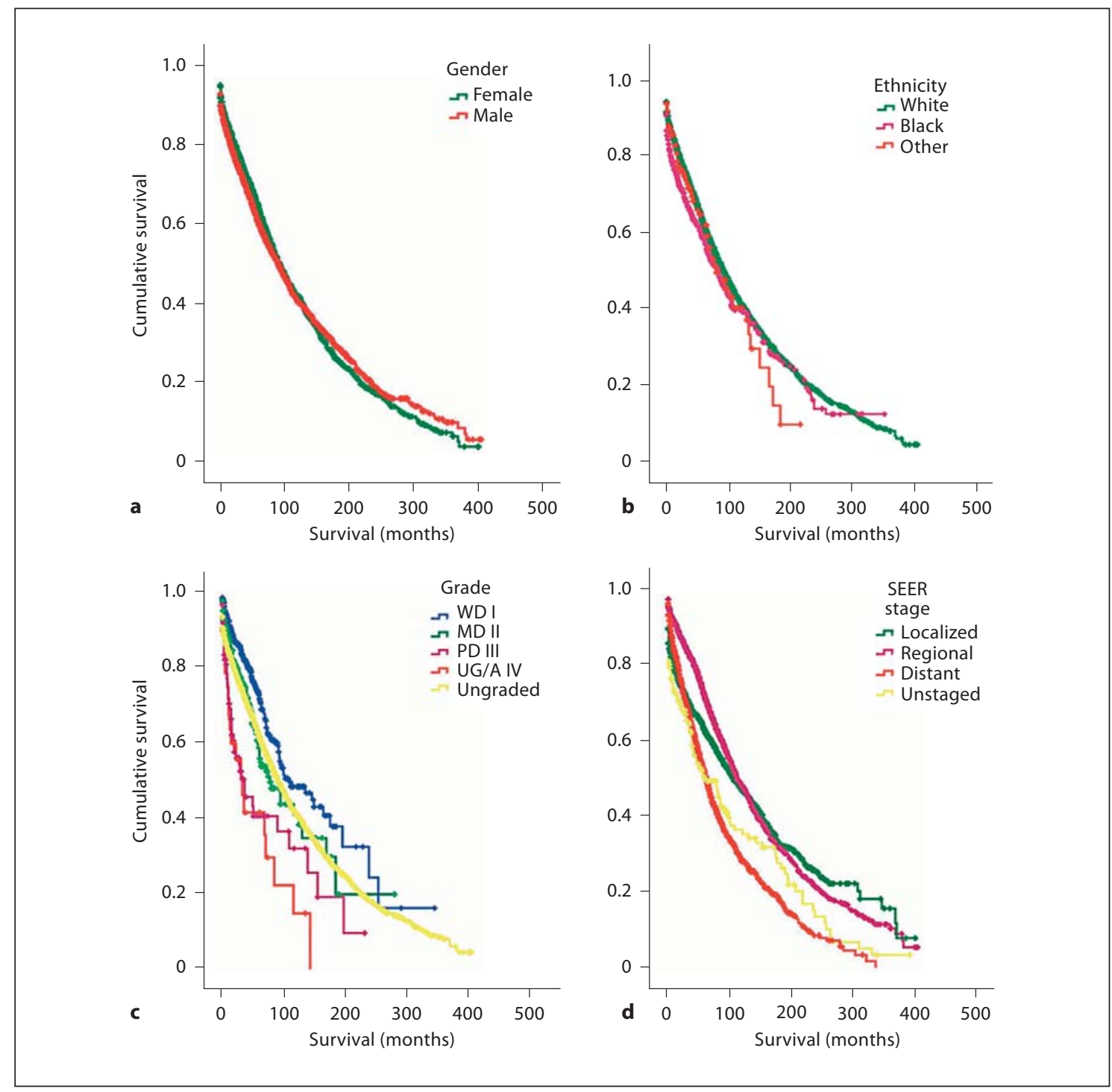

Fig. 1. SEER database analysis 2007. Survival by gender (a), race (ethnicity, b), histological grade (c) and SEER staging (extent, d) of $\mathrm{n}=7,445$ patients. A significant survival effect was conferred by grade, stage and ethnicity but not gender. WD = Well-differentiated (grade I); $\mathrm{MD}=$ moderately differentiated (grade II); $\mathrm{PD}=$ poorly differentiated (grade III); UG/A = undifferentiated/anaplastic (grade IV). SEER stage abbreviations refer to localized, regional lymph node involvement or distant metastases.

\section{Results}

HRs for the prognostic impact of each variable were obtained from the SEER database and from literature review.

\section{De novo Analysis of the SEER Database}

Clinicopathological characteristics, survival (in months) and analysis of factors (HRs) associated with survival of the NCI SEER patient population are included in table 4; survival curves are displayed in figure 1. Significant effects of ethnicity, tumor histology and dissemination (localized, regional or distant) were associated with risk of death. These data were included in the nomogram (table 5; fig. 2).

\section{Prognostic Variables in the NET Literature}

Age. Increasing age, particularly high age, represents a risk factor for poor prognosis in most cancers [35]. Com- 


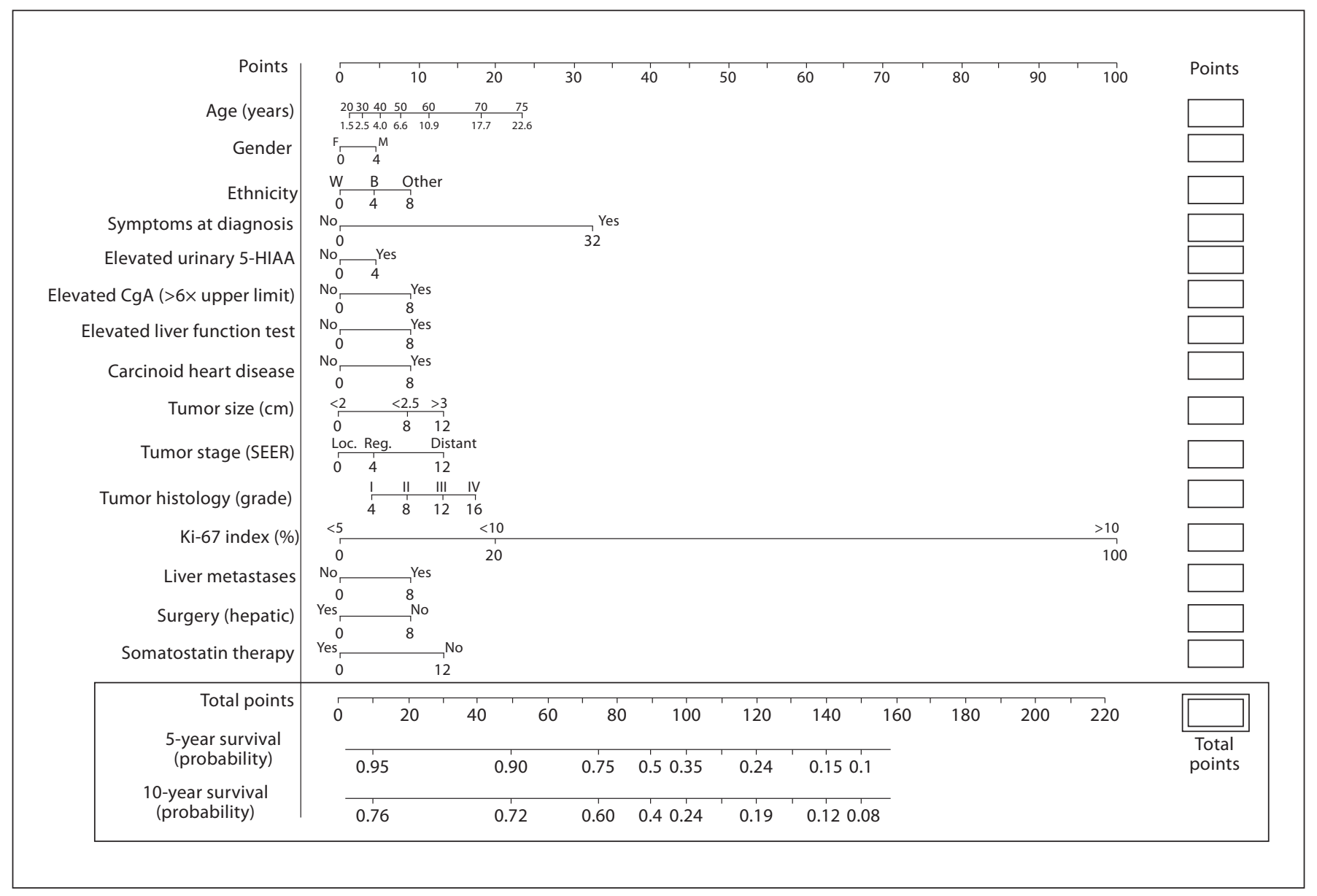

Using the nomogram

- Age is the only continuous variable - all others are categorical

- 'Ethnicity' is based on SEER data, and therefore the SEER specificity criteria are retained such that 'other' includes Hispanic and Latino

- 'Symptoms at diagnosis' refers to carcinoid syndrome

- 'Elevated urinary 5-HIAA' reflects any elevation outside the normal range

- 'Elevated liver function tests' refers to any elevation beyond the normal range in bilirubin, alkaline phosphatase or $\gamma$-glutamyltransferase

- 'Carcinoid heart disease' requires echocardiographic assessment and confirmation

- 'SEER stage' abbreviations refer to localized (loc.), regional lymph node involvement (reg.) or distant metastases

- 'Tumor grade': I = well differentiated; II = moderately differentiated; III = poorly differentiated; IV = undifferentiated/ anaplastic

- 'Liver metastases' is a clinical designation based upon available imaging modalities

Fig. 2. Five- and 10-year probability survival nomogram for SI NETs based on the overall literature review $(\mathrm{n}=$ $12,412)$ and additional analysis of 7,445 patients in the NCI SEER database. $\mathrm{F}=$ Female; $\mathrm{M}=$ male; $\mathrm{W}=\mathrm{White}$; $\mathrm{B}=$ Black; 5-HIAA = 5-hydroxyindoleacetic acid; CgA = chromogranin A. 
Fig. 3. NET nomoscores and survival analysis (Kaplan-Meier) in the 3-group, international validation sample $(\mathrm{n}=33)$. a Nomogram scores were significantly increased ( $\mathrm{p}=0.013$, Mann-Whitney, 2tailed) in deceased $(n=17)$ compared to living patients $(n=16)$. b Survival analysis using the Kaplan-Meier approach identified that a nomogram score $<75$ (group 1) conferred a mean survival of $15.5 \pm 4.3$ years. Group 2 (nomoscore: 75-95) and group 3 (nomoscore $>95$ ) had significantly lower survivals $(9.7 \pm 2.4$ and $6.4 \pm 1.1$ years, respectively; $\mathrm{p}=0.032, \log$ rank test).

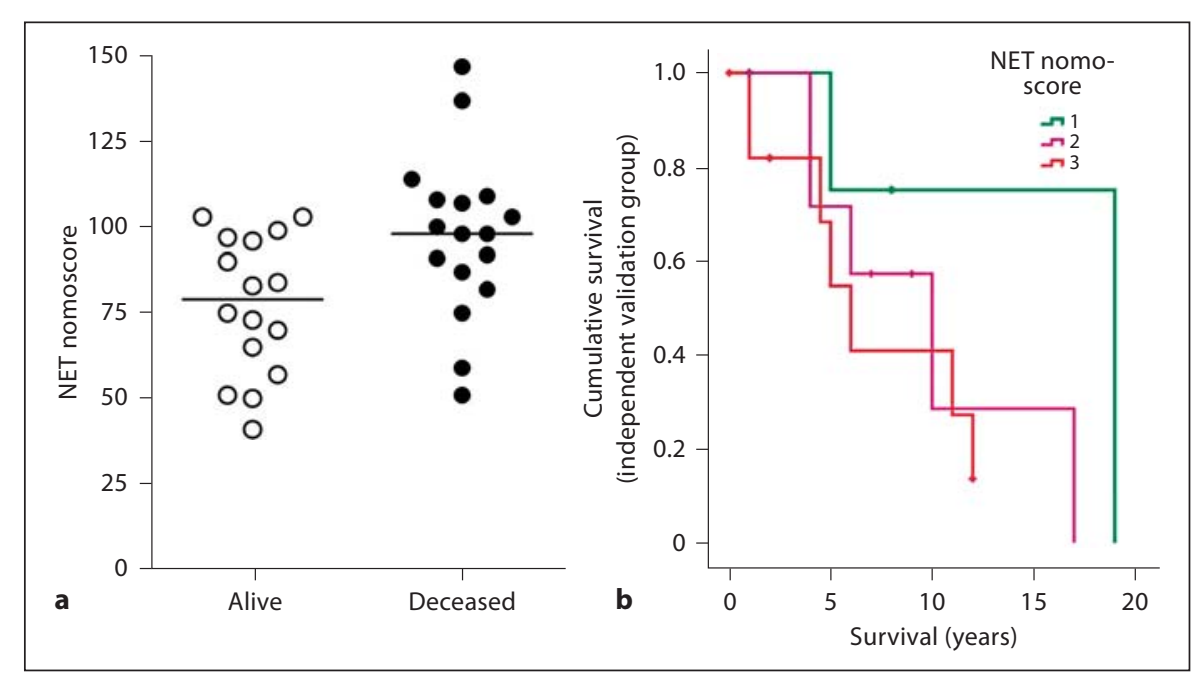

pared to GEP NET patients $<65$ years, the excess 5 -year death risk for patients $>65$ years is doubled (1:1.91) [20]. Among 258 SI NET patients, age $\geq 64$ years demonstrated a significantly increased risk of death with a univariate analysis HR of 2.78 and a multivariate analysis HR of 3.12 [14]. An Italian study of 156 GEP NETs (67 pancreatic endocrine tumors, 73 gastrointestinal carcinoids and 16 of unknown origin) noted that age $>50$ years compared to $<50$ years at diagnosis correlated with an increased risk of death, HR 2.33 (univariate analysis) [21]. Similarly, in a Dutch study of 76 patients with midgut carcinoid tumors, age as a continuous variable was a prognostic factor for survival with an HR of 1.043 and 1.052 per year for uni- and multivariate analyses, respectively [23]. A large European multicenter study comprising 7,693 patients with GEP NETs investigated the relative risk of death for patients $\geq 75$ years and the age group of $55-74$ years, and identified that compared to patients between 15 and 54 years these two groups exhibited HR values of 3.58 and 2.26, respectively [18]. A report from the Mayo Clinic examined long-term survival of gastrointestinal carcinoids ( $n=154$, median age 62 years, range $12-84$ years) classified as foregut (7\%), midgut (62\%) and hindgut (30\%) origin [33]. Overall, the HR in multivariate analysis for death in patients $>62$ years was 3.40 , and the HR was 2.7 in the subgroup classified as midgut carcinoids [33]. Among 3,231 well-differentiated GEP NETs in England and Wales, patients aged 55-74 years had an HR of 1.9 for death compared to the group aged 15-54 years [20].

Gender. Male gender represents an independent risk factor for decreased 5-year survival in GEP NET disease. In a European multicenter study including 3,715 GEP NETs, 5 -year survival was $45.5 \%$ for men compared to

Nomogram to Assess Small-Intestinal

Neuroendocrine Tumor Survival
49.4\% for women, and the odds ratio for death was 1:0.89 [20]. In a study assessing gender in England and Wales (1986-2001), in 3,231 well-differentiated GEP NETs, the HR for death for women was 0.8 compared to men [20]. Similarly, in an analysis of a Scandinavian cohort of 258 SI NETs, male patients had a significantly increased risk of death, with an HR of 1.66 in univariate analysis which was similar (1.52) but did not reach statistical significance in multivariate analysis [14].

Ethnicity. Race is a well-recognized independent risk factor for survival $[11,12]$. In the USA, Asians have a better prognosis and Blacks a slightly worse prognosis, compared to Whites. Specifically, Asians have the best survival among patients with localized disease, whereas Whites had the best survival among patients with metastatic disease $[11,12]$.

Clinical Symptomatology (Any Clinical Symptom Related to the Neoplasm, Hormonal or Nonhormonal). In a mixed NET population $(\mathrm{n}=399$; foregut $46.1 \%$, midgut $37.1 \%$, hindgut $4.5 \%$, unknown origin $10.5 \%$ ), multivariate analysis demonstrated an HR of 8.2 for death (5-year survival) if clinical symptoms were present at diagnosis [22].

Hormonal Symptoms. Tumors that produce bioactive products leading to the carcinoid syndrome have been associated with a worse prognosis with an HR of 2.9 (yes vs. no) for shorter 5-year survival [19]. Presumably this may reflect not only the production of bioactive products, but also the presence of hepatic metastases or carcinoid heart disease.

Urinary 5-HIAA. Among 256 patients with midgut carcinoid tumors, urinary 5 -HIAA $>300 \mu \mathrm{mol} / 24 \mathrm{~h}$ was associated with a shorter 5 -year survival, 45 versus 72 
months, and an HR of death of 1.8 compared to those with values $<300 \mu \mathrm{mol} / 24 \mathrm{~h}$ [19]. Another study that assessed 90 metastatic GEP NETs found similar results with an HR of 2.36 for death in multivariate analysis if urinary 5-HIAA levels were increased twice the upper normal limit [17]. An evaluation of 258 SI NETs demonstrated that a urinary 5 -HIAA ratio $>3.7 \times$ the upper normal limit was associated with an $\mathrm{HR}$ of 2.35 in univariate analysis but this factor was nonsignificant in multivariate analysis [14].

Chromogranin A Blood Levels. In a study of 301 patients with carcinoid tumors, plasma chromogranin A values $>5,000 \mu \mathrm{g} / \mathrm{l}$ were associated with a shorter 5 -year survival, 33 versus 57 months, and an HR of death of 4.5 compared to those with values $<5,000 \mu \mathrm{g} / \mathrm{l}$ [19]. A Norwegian retrospective study of 258 SI NETs found that a chromogranin A ratio $>6.2 \times$ the upper limit of normal was associated with an HR of 2.47 in univariate analysis and 1.90 in multivariate analysis [14].

Abnormal Parameters of Hepatic Function. In a retrospective analysis of 137 patients with metastatic NETs, alkaline phosphatase levels above normal were associated with excess risk of death (HR 2.4), compared to patients with normal alkaline phosphatase levels [15]. Similarly, 21 metastatic GEP NETs with elevated liver function tests (alkaline phosphatase, bilirubin and $\gamma$-glutamyltransferase), exhibited an HR of 2.08 of death compared to 84 patients with normal values [17].

Carcinoid Heart Disease. This represents a serious directly related comorbidity of serotonin-producing NETs [36]. The presence of carcinoid heart disease represents an individual risk factor for death in SI NET disease, with an HR of 1.76 in univariate analysis [14]. Among the subgroup of patients with the carcinoid syndrome, echocardiography can be used to further predict the 5-year survival [28]. The HR for death in 52 patients with the carcinoid syndrome was 2.52 (univariate analysis) if tricuspid regurgitation was present [28].

Tumor Size. As indicated in the TNM classification, increasing tumor size has intuitively been associated with outcome in many forms of neoplasia [37]. Evaluating a heterogeneous group of NETs $(n=399$; foregut $46.1 \%$, midgut $37.1 \%$, hindgut $4.5 \%$, unknown origin $10.5 \%$ ) and using a multivariate analysis, Pape et al. [34] demonstrated an HR of 4.44 for death (5-year survival) if the size of the primary tumor was $>2.5 \mathrm{~cm}$. Similarly, Panzuto et al. [21] reported that in 156 GEP NETs (67 pancreatic endocrine tumors, 73 gastrointestinal carcinoids and 16 of unknown origin) a primary tumor size of $>3 \mathrm{~cm}$ increased the risk of death with an HR of 4.26 (univariate analysis).
Metastasis. Although the recognition that spread beyond the primary location is associated with diminished outcome, the precise relationship between the extent (tumor volume) and location of spread (local area, liver, lymph node and extracompartmental disease) has been difficult to quantify. In 256 patients with midgut carcinoid tumors, individuals with local disease (primary tumor and/or lymph node metastases) exhibited a median survival of 108 months, compared to 159 months if $<5$ liver metastases and 53 months if $>5$ liver metastases [19]. Thus, hepatic metastasis represented a critical variable. Similarly, the extent of the hepatic metastases was notable in that the HR for $>5$ liver metastases compared to no metastases was 3.0 [19]. Retrospective investigation of 258 SI NETs found that liver or other distant metastasis was associated with an HR of 2.70 in univariate analysis and 1.98 in multivariate analysis [14]. Yet another study of tumors classified as midgut NETs (77 SI, 17 appendix and 1 right colon) noted that the presence of liver metastases at diagnosis correlated with an HR of 2.3 for excess death risk (multivariate analysis) [33].

Histological Morphological Grade. Among factors that impact the prognosis for GEP NETs, differentiation represents one of the most critical determinants in most studies. In a survival analysis that collated data from the cancer registries of 12 European countries, a 4 -fold increase in relative excess risk of death within 5 years of diagnosis for poorly differentiated NETs compared to well-differentiated ones (HR 1.0:0.27) was identified [20]. Similar results were demonstrated in a study of 119 metastatic NETs in which poorly differentiated tumors had an increased relative risk of death with an HR of 4.02 compared to well-differentiated tumors [17]. Panzuto et al. [21] reported that in 156 GEP NETs (67 pancreatic endocrine tumors, 73 gastrointestinal carcinoids and 16 of unknown origin) being poorly versus well differentiated was associated with an increased risk of death, with HR 7.70 (univariate analysis) and 2.99 (multivariate). In a large European multicenter study that included 7,693 (6,718 well differentiated and 975 undifferentiated), the 5 -year survival for well-differentiated lesions was $62.1 \%$ in contrast to undifferentiated tumors (7.8\%) while the HR of death was 4.60 compared to those classified as well differentiated [18].

$\mathrm{Ki}-67$ Index. Ki-67 is a marker of the proliferative activity in neoplasia and is regarded by many neuroendocrine authorities as a critical index in determining both outcome and therapy, although there is some disagreement as to how it should be calculated [10]. Cunningham et al. [16] examined the clinical relevance of Ki-67 in 81 
midgut carcinoid patients all of whom had metastases with a survival range of 1-223 months. Primary tumors with a Ki-67 index $\geq 1.0 \%$ had an HR for death of 5.4 compared to those with Ki-67 $<1 \%$ [16]. When metastases were investigated, a Ki-67 index $\geq 1.0 \%$ had an HR for death of 2.5 compared to those with Ki-67 $<1 \%$ [16]. Bergestuen et al. [14] reported that in 130 SI NETs, Ki-67 values of $\geq 5 \%$ were associated with a significantly increased risk of death (HR of 2.24) in univariate analysis. Separate from the prior two Scandinavian studies, a study of a German mixed NET population $(\mathrm{n}=399$; foregut $46.1 \%$, midgut $37.1 \%$, hindgut $4.5 \%$, unknown origin $10.5 \%$ ) demonstrated an HR of 3.99 (multivariate analysis) for death (5-year survival) if the Ki-67 index was between 5 and $10 \%$ and an HR of 24.8 if Ki-67 was $>10 \%$ [34]. An Italian study of 156 GEP NETs (67 pancreatic endocrine tumors, 73 gastrointestinal carcinoids and 16 of unknown origin) found that if the Ki-67 index was $>2$, the risk of death was increased with an HR of 3.84 (univariate analysis) [21].

\section{Extrapolation of HRs to the Prognostic Model}

The HR for each variable, developed in multivariate models in previous prognostic studies or de novo from the NCI SEER database, was translated directly into a prognostic weighting (table 5). For example, the HR for symptoms at diagnosis was 8.2 and was therefore assigned a weighting of 8 points, which, like all variables, was then multiplied by 4 to achieve the final score (variable: symptoms at diagnosis, score 32).

\section{Internal Validation}

The nomogram was applied to a validation sample comprising 33 patients with SI NETs from the 3 collaborating institutions. An examination of nomogram scores identified significant elevation $(\mathrm{p}=0.01)$ in deceased compared to living patients (fig. 3a). For Kaplan-Meier analyses, scores were stratified into 3 different groups by 5-/10-year survival: group 1 , nomoscore $<75$ (estimated 5 -year survival $>0.75, \mathrm{n}=10$ ); group 2 , nomoscore 75-95 (estimated 5-year survival 0.4-0.74, $\mathrm{n}=9$ ), and group 3, nomoscore $>95$ (estimated 5-year survival $<0.4, \mathrm{n}=14$ ). At the time of analysis $80 \%$ of group $1,37.5 \%$ of group 2 and $31.2 \%$ of group 3 were alive. The estimated mean survival of group 1 was $15.5 \pm 4.3$ years, for group 2 it was $9.7 \pm 2.5$ years and $6.4 \pm 1.1$ years for group 3 (table 6 ). This was statistically significant $\left(\chi^{2}=4.6, p=0.03, \log \right.$ rank test) and indicated that the nomogram score effectively predicted survival (fig. 3b).

Nomogram to Assess Small-Intestinal Neuroendocrine Tumor Survival
Table 6. Summary of Kaplan-Meier analysis of NET nomogram scores

\begin{tabular}{llllll}
\hline $\begin{array}{l}\text { NET } \\
\text { nomo- } \\
\text { score }\end{array}$ & Group & Risk & $\begin{array}{l}\text { Mean } \\
\text { follow-up } \\
\text { years }\end{array}$ & $\begin{array}{l}\text { Per- } \\
\text { centage } \\
\text { alive }\end{array}$ & $\begin{array}{l}\text { Mean } \\
\text { survival } \\
\text { years }\end{array}$ \\
\hline$<75$ & 1 & low & $5.33 \pm 5.8$ & 80 & $\begin{array}{r}15.5 \pm 4.3 \\
75-95\end{array}$ \\
$>95$ & 3 & medium & $6.61 \pm 4.9$ & 37.5 & $9.7 \pm 2.5$ \\
& high & $4.6 \pm 4.2$ & 31.2 & $6.4 \pm 1.1$ \\
\hline
\end{tabular}

${ }^{1}$ At time of current assessment (June 2010).

\section{Discussion}

At present, the criteria for assessing the prognosis and predicting the progression of SI NETs represent a variety of clinical and pathological indices that are adjudged differently in various countries and interpreted variably by different caregivers. There exists no mathematically based assessment of a compilation of clinical, pathological and biochemical parameters to provide a multivariate assessment of the weighting of the numerous different indices that comprise the clinical milieu within which the individual disease of a particular patient and tumor may be objectively assessed. This NET nomogram (Modlin Score) provides a tool that can be utilized for prognosis prediction, patient group comparisons and serve as a guide for stratification of treatment and surveillance. Not least is the fact that a patient may be provided with an objective assessment by a physician regarding the future course of his disease, thereby helping to allay a critical concern of many patients as to "what the future may hold for them'.

The nomogram is useful for visualizing the associations between each predictor variable and SI NET-specific death. However, there are limitations given the paucity of data that can be evaluated for some indices as well as the future need for the identification of specific biomarkers that define the proliferative capacity of NET cells and identify metastatic potential. In addition, independent external validation is necessary to confirm efficacy and identify possible additional indices that might strengthen the mathematical basis of prediction. The predictive power of the nomogram in the current validation sample is statistically significant but modest, reflecting the limited size of the validation sample. As the nomogram is tested in other sample sets, we expect some modification and substantial improvement in its predictive power.

Neuroendocrinology 2010;92:143-157 153 
Nevertheless, this nomogram represents an objective analysis of all currently available data, a statistical assessment of its accuracy and an analytic compilation of its numeric efficacy in predicting survival. As such it provides a comprehensive and easily utilized clinical tool whereby SI NET disease may be objectively assessed and applied to patient management. No such instrument currently exists and it is likely that application of this nomogram will yield further data that can be added to it and allow for greater accuracy and increased predictive ability. The overall utility of this approach is illustrated in figure 3. A random sample of US and European patients from three different institutions identified that assessment of the proposed indices and calculation of a NET nomoscore accurately identified patients at low ( $<75$ points), medium (75-95 points) and high ( $>95$ points) risk of death.

In the examination of the nomogram predictors, there is an inherent difficulty in studying one predictor variable at a time since some predictors are often correlated; thus, moving a patient on one axis may tend to simultaneously alter a related axis. Changing clinical criteria are such that altering one variable while holding all others fixed may therefore not accurately reflect the mathematical function of the predictor axes. It is likely that the calculation of the nomogram at fixed time points may therefore also be able to provide a geometric construct predicting disease advance or stabilization as denominator of index increase or decrease calculated as a patient curve slope against that of a cumulative value derived from a large cohort of comparable tumors. Although we have used a point system nomogram in figure 2 , an alternative representation would be a table. However, a table would require categorization of the continuous variable 'age', thereby reducing predictive accuracy. In addition, a table with all possible combinations of the predictor variable values might be cumbersome. A different option for the nomogram could be in the form of survival curves. However, this representation suffers from the same limitations as the table. The advantage of the point system is that it preserves continuous variables and accuracy in an efficient manner. Although our results do not represent definitive comparisons of all alternative techniques, nor can it necessarily be concluded that Cox proportional modeling and hazard analysis are the best tools for prediction, the analysis does provide the platform on which a predictive nomogram can be utilized or further developed. Subsequent validated iterations might limit the number of predictive variables in the score, for example, but at this time the best model required all the variables described. A possible extrapolation of a nomogram of this kind can be the development of an electronic version for a handheld software application, minimizing computational burden and allowing for easy clinic or bedside prediction as well as the development of additional prediction time points (e.g. 1- to 20-year predictions; table 7).

The nomogram may be useful for patient counseling, because it predicts the probability that the patient will die of SI NET disease within 5-10 years of diagnosis and treatment, assuming death does not occur for other reasons. Similarly, patients who are anxious since they believe they are at high risk of tumor-related death may be reassured based upon their nomogram score (table 7). Physicians who are unable to determine patients at high risk of death from SI NETs can use the tool to identify patients appropriate for early interventional therapy. A clear advantage of the nomogram is that it can be used to predict disease-specific death more accurately than would be achieved with straightforward subset analysis with the Kaplan-Meier method. The nomogram could be used to identify patients by computing their probability of SI NET-specific death at 5 and 10 years, followed by offering the therapy to those whose prediction is higher than a predetermined amount, which is treatment dependent.

Older patients will likely have a higher NET-specific death prediction than younger patients. Histology seems to be an important predictor, extending across the full range of the point axis. In addition, the nomogram illustrates the magnitude of the worsening prognosis as the tumor size increases. The nomogram clearly identifies the shift in prognosis associated with grade of the tumor and especially the Ki-67 index. Thus, for example, individuals with low-grade metastatic histological disease plus minimal other indices of an adverse nature might have a low to intermediate death prediction score. This would be amended to a substantially higher death prediction if the histological parameters changed to high-grade disease. Nevertheless, the proposed numerical weighting system is based on published data and may be objectively revised once additional prospective histomorphological data are assimilated into the nomogram and further external validation is undertaken.

Additionally, the nomogram may become useful in determining the interval necessary for ideal follow-up visits, since patients at lower risk for NET-specific death may require less stringent follow-up evaluation. The nomogram can be used as a potential method to measure the efficacy of individual therapies such as surgery or a particular therapy by assessment of outcome alteration in comparison with a similar nontreated group. In addition, such calculations would allow for the development of economic 
assessments of cost-effectiveness of a particular management strategy among comparable disease groups. Ultimately, predictions from the nomogram could be used as probabilities in a mathematically derived decision analytic model. The role of therapy for NETs remains controversial, unpredictable and incompletely defined for a number of reasons including inadequate ability to type tumors at a molecular level, limitations in tumor and patient stratification and a paucity of identifiable targeted therapeutic options, e.g. transduction pathway identified but no evidence of its presence in a particular patient tumor $[2,38]$. Consequently clinical strategies have been inconclusive or have yielded only modest data with minimal benefit. In most instances, results have been confounded by the inclusion of different types of NETs, a variety of staging/grading systems and underpowered studies as well as a wide range in survival within the WHO/AJCC/ ENETS classification compilation. Using the nomogram, we have identified that patients entered into clinical trials could be more stringently stratified.

There are some areas that will require adjustment in the future. In particular, the pathological classifications used in the NCI SEER database are not used uniformly in Europe where neither a classification with 'moderately differentiated NET', separate from well-differentiated neuroendocrine carcinoma or anaplastic as a separate group from poorly differentiated NET, is utilized. Once an external validation is undertaken, it might be necessary to consider 'poorly differentiated' and 'anaplastic' as one group on the scale unless there are sufficient data to support the separation. Similarly, the importance of the histological grade is considered on the basis of the differences between well- and poorly differentiated SI NETs while it might be argued that the use of 4 grades for this scale adds variables for which it is difficult to ensure uniform objectivity. Possibly the use of only 2 - 'well' and 'poorly differentiated' - may be worthy of consideration given the unlikelihood that a globally acceptable pathological classification will become available in the discernible future or reconsidered once data from the WHO classification 2011 are available. A key unmet need and criticallimitation in the development of this nomogram is the absence of any molecular predictors of tumor behavior and the potential for metastasis.

There is, in some areas of the nomogram, apparent discordance with clinical intuition and anecdotal experience. Thus, the discrimination of histology (well vs. poorly differentiated), as well as the observation that the overall score for grades III and IV only accrues 12 and 16 points which appears underestimated in comparison to
Table 7. Summary of potential utilities of a NET nomogram

Predict prognosis

- At diagnosis

- To assess progression

- Reevaluation following treatment

Patient counseling

- Provide objective layman-assessable information

- Information to family

- Objective information for insurance company assessment

Formalize patient stratification

- High-risk versus low-risk groups

- Treatment versus no treatment

- Early intervention or expectant strategy

- Surveillance intervals

Evaluate treatment efficacy

- Surgical

- Medical

- Peptide radioreceptor therapy

- Ablation (embolization/radiofrequency)

Guide to therapeutic strategy

- Poor prognosis/aggressive treatment

- When to initiate treatment

- When to cease/alter treatment

Use in hand-held computers

- Facilitate assessment in oncology clinic

- Objective information for nonspecialist MD

Research tool

- More stringent/comparable stratification of clinical trial patients

- Assess outcome for individual therapy or treatment versus no treatment

- Patient group comparisons

score ratios applied to symptoms. Similarly, a poorly differentiated or anaplastic delineation (median survival ranging from 10 to 77 months, depending on the database and method of analysis) seems to be an underestimated prognostic factor (12-16 points in the current nomogram) compared to Ki-67 >10\% (100 points). Nevertheless, these assessments are based upon available data. It is likely that such inconsistencies may become refined or mathematically reworked with the prospective evaluation of an external database.

Several additional variables may provide potentially useful prognostic information but these were not included in the nomogram since, at present, such data or technology is not available in all clinical centers. The patient's geographical location has been explored in some European regions and this feature significantly influences survival $[2,18,20,39,40]$. Somatostatin receptor positiv- 
ity on Octreoscan or ${ }^{68} \mathrm{Ga}$-DOTATOC positron emission/computed tomography imaging is associated with better survival [41-43], and ${ }^{18} \mathrm{~F}$-fluorodesoxyglucose positron emission/computed tomography positivity is linked to a poorer outcome [44]. As sophisticated scanning technology becomes more widely accessible, these additional parameters may be added and further amplify the accuracy of the nomogram. Similarly, the inclusion of novel molecular indices of proliferation and metastasis may also become available and provide increasingly accurate prognostication. The nomogram therefore, by definition, serves as a developmental framework for the evolution of prognostication.

Overall, the nomogram has limitations because some factors impacting survival are incompletely characterized at present. However, by taking into account a greater number of known factors, a survival nomogram allows for a more realistic approximation of whether an individual patient will be alive for a defined period of time. Longer follow-up, more patients and novel predictors are likely to improve nomogram accuracy. The decision to model NET-specific survival, which is hypothetical, rather than overall survival, is debatable; however, the addition of death from other causes would warrant inclusion of several other predictors (e.g. comorbidity and socioeconomic status) to avoid assuming that patients with the same disease-specific covariate values also have the same risk of death from other causes. We have therefore eschewed this strategy and chosen to focus on NET disease variables. In the meantime, it is conceivable that the no- mogram may provide the most accurate predictions presently available. By identifying a group with a more homogeneous prognosis, the interpretation of trial outcomes may become clearer.

The purpose of assembling this nomogram was to provide a clinical tool that is objective, assesses a diverse range of parameters and is globally applicable by physicians to predict the prognosis of small-bowel NETs (table 7). It can also be used to assess outcome and the potential of NET-specific treatment to modify the disease end point as well as allow for internationally comparable patient and tumor stratification. As the biology of NET disease is further elucidated, additional clinical, pathological and molecular markers can be validated and incorporated to amplify the predictive value and accuracy of this clinical tool. With the availability of external validation, one may anticipate advances in physician decision making, individual patient counseling and the implementation of rational therapeutic strategies based upon quantifiable parameters that can be assessed and compared on a global basis.

\section{Acknowledgments}

Henning Jann of the Charité University and Ms. Maria Theresia Svejda of Yale University are acknowledged for collection and assessment of patient data. We thank Prof. Michael Krauthammer, of Yale University School of Medicine, for his insightful overview and sagacious commentary regarding biostatistical aspects of the paper.

\section{References}

1 Modlin I, Shapiro M, Kidd M: Siegfried Oberndorfer - origins and perspectives of carcinoid tumors. Hum Pathol 2004;35: 1440-1451.

2 Modlin IM, Oberg K, Chung DC, Jensen RT, de Herder WW, Thakker RV, Caplin M, Delle Fave G, Kaltsas GA, Krenning EP, Moss SF, Nilsson O, Rindi G, Salazar R, Ruszniewski P, Sundin A: Gastroenteropancreatic neuroendocrine tumours. Lancet Oncol 2008;9: 61-72.

-3 Modlin IM, Champaneria MC, Bornschein J, Kidd M: Evolution of the diffuse neuroendocrine system - clear cells and cloudy origins. Neuroendocrinology 2006;84:69-82.

4 Modlin I, Lye K, Kidd M: A five-decade analysis of 13,715 carcinoid tumors. Cancer 2003;97:934-959.
5 Kloppel G, Perren A, Heitz PU: The gastroenteropancreatic neuroendocrine cell system and its tumors: the WHO classification. Ann NY Acad Sci 2004;1014:13-27.

6 Greene FL: TNM staging for malignancies of the digestive tract: 2003 changes and beyond. Semin Surg Oncol 2003;21:23-29.

7 Kloppel G, Rindi G, Perren A, Komminoth P, Klimstra DS: The ENETS and AJCC/ UICC TNM classifications of the neuroendocrine tumors of the gastrointestinal tract and the pancreas: a statement. Virchows Arch 2010;456:595-597.

-8 Washington MK, Tang LH, Berlin J, Branton PA, Burgart LJ, Carter DK, Compton CC, Fitzgibbons PL, Frankel WL, Jessup JM, Kakar S, Minsky B, Nakhleh RE: Protocol for the examination of specimens from patients with neuroendocrine tumors (carcinoid tumors) of the small intestine and ampulla. Arch Pathol Lab Med 2009;134:181-186.
9 Rindi G, Kloppel G, Couvelard A, Komminoth P, Korner M, Lopes J, McNicol A, Nilsson O, Perren A, Scarpa A, Scoazec J, Wiedenmann B: TNM staging of midgut and hindgut (neuro)endocrine tumors: a consensus proposal including a grading system. Virchows Arch 2007;451:757-762.

10 Klimstra D, Modlin I, Adsay N, Chetty R, Deshpande V, Gonen M, Jensen R, Kidd M, Kulke MRL, Moran C, Moss S, Oberg K, O’Toole D, Rindi G, Robert M, Suster S, Tang L, Tzen CY, Washington M, Wiedenmann B, Yao J: Pathologic reporting of neuroendocrine tumors: application of the Delphic consensus process to the development of a minimum pathologic data set. Am J Pathol 2010; 34:300-313. 
11 US National Cancer Institute: Surveillance Epidemiology and End Results (SEER) data base. 1973-2007.

- 12 Yao JC, Hassan M, Phan A, Dagohoy C, Leary C, Mares JE, Abdalla EK, Fleming JB, Vauthey JN, Rashid A, Evans DB: One hundred years after 'carcinoid': epidemiology of and prognostic factors for neuroendocrine tumors in 35,825 cases in the United States. J Clin Oncol 2008;26:3063-3072.

13 Modlin IM, Moss SF, Chung DC, Jensen RT, Snyderwine E: Priorities for improving the management of gastroenteropancreatic neuroendocrine tumors. J Natl Cancer Inst 2008;100:1282-1289.

$\checkmark 14$ Bergestuen DS, Aabakken L, Holm K, Vatn $M$, Thiis-Evensen E: Small intestinal neuroendocrine tumors: prognostic factors and survival. Scand J Gastroenterol 2009;44: 1084-1091.

- 15 Clancy TE, Sengupta TP, Paulus J, Ahmed F, Duh MS, Kulke MH: Alkaline phosphatase predicts survival in patients with metastatic neuroendocrine tumors. Dig Dis Sci 2006; 51:877-884.

16 Cunningham JL, Grimelius L, Sundin A, Agarwal S, Janson ET: Malignant ileocaecal serotonin-producing carcinoid tumours: the presence of a solid growth pattern and/or Ki67 index above $1 \%$ identifies patients with a poorer prognosis. Acta Oncol 2007;46:747756.

-17 Formica V, Wotherspoon A, Cunningham D, Norman AR, Sirohi B, Oates J, Chong G: The prognostic role of WHO classification, urinary 5-hydroxyindoleacetic acid and liver function tests in metastatic neuroendocrine carcinomas of the gastroenteropancreatic tract. Br J Cancer 2007;96:1178-1182.

- 18 Gatta G, Ciccolallo L, Kunkler I, Capocaccia R, Berrino F, Coleman MP, De Angelis R, Faivre J, Lutz JM, Martinez C, Moller T, Sankila R: Survival from rare cancer in adults: a population-based study. Lancet Oncol 2006;7:132-140.

19 Janson ET, Holmberg L, Stridsberg M, Eriksson B, Theodorsson E, Wilander E, Oberg K: Carcinoid tumors: analysis of prognostic factors and survival in 301 patients from a referral center. Ann Oncol 1997;8:685-690.

20 Lepage C, Rachet B, Coleman MP: Survival from malignant digestive endocrine tumors in England and Wales: a population-based study. Gastroenterology 2007;132:899-904.

21 Panzuto F, Nasoni S, Falconi M, Corleto VD, Capurso G, Cassetta S, Di Fonzo M, Tornatore V, Milione M, Angeletti S, Cattaruzza MS, Ziparo V, Bordi C, Pederzoli P, Delle Fave G: Prognostic factors and survival in endocrine tumor patients: comparison between gastrointestinal and pancreatic localization. Endocr Relat Cancer 2005; 12:10831092.
22 Pape UF, Jann H, Muller-Nordhorn J, Bockelbrink A, Berndt U, Willich SN, Koch M, Rocken C, Rindi G, Wiedenmann B: Prognostic relevance of a novel TNM classification system for upper gastroenteropancreatic neuroendocrine tumors. Cancer 2008; 113:256-265.

23 Van der Horst-Schrivers AN, Post WJ, Kema IP, Links TP, Willemse PH, Wymenga AN, de Vries EG: Persistent low urinary excretion of 5-HIAA is a marker for favourable survival during follow-up in patients with disseminated midgut carcinoid tumours. Eur J Cancer 2007;43:2651-2657.

24 Iasonos A, Schrag D, Raj GV, Panageas KS: How to build and interpret a nomogram for cancer prognosis. J Clin Oncol 2008;26: 1364-1370.

25 Kattan MW, Leung DH, Brennan MF: Postoperative nomogram for 12-year sarcomaspecific death. J Clin Oncol 2002;20:791796.

26 Landry CS, Brock G, Scoggins CR, McMasters KM, Martin RC 2nd: A proposed staging system for small bowel carcinoid tumors based on an analysis of 6,380 patients. Am J Surg 2008;196:896-903, discussion 903.

27 Cadden I, Johnston BT, Turner G, McCance D, Ardill J, McGinty A: An evaluation of cyclooxygenase-2 as a prognostic biomarker in mid-gut carcinoid tumours. Neuroendocrinology 2007;86:104-111.

28 Westberg G, Wangberg B, Ahlman H, Bergh $\mathrm{CH}$, Beckman-Suurkula M, Caidahl K: Prediction of prognosis by echocardiography in patients with midgut carcinoid syndrome. Br J Surg 2001;88:865-872.

-29 Rinke A, Muller HH, Schade-Brittinger C, Klose KJ, Barth P, Wied M, Mayer C, Aminossadati B, Pape UF, Blaker M, Harder J, Arnold C, Gress T, Arnold R: Placebo-controlled, double-blind, prospective, randomized study on the effect of octreotide LAR in the control of tumor growth in patients with metastatic neuroendocrine midgut tumors: a report from the PROMID Study Group. J Clin Oncol 2009;27:4656-4663.

30 Bernheim AM, Connolly HM, Rubin J, Moller JE, Scott CG, Nagorney DM, Pellikka PA: Role of hepatic resection for patients with carcinoid heart disease. Mayo Clin Proc 2008;83:143-150

31 Townsend A, Price T, Yeend S, Pittman K, Patterson K, Luke C: Metastatic carcinoid tumor: changing patterns of care over two decades. J Clin Gastroenterol 2010;44:195199.

32 Moller JE, Pellikka PA, Bernheim AM, Schaff HV, Rubin J, Connolly HM: Prognosis of carcinoid heart disease: analysis of 200 cases over two decades. Circulation 2005; 112:3320-3327.
3 Soreide JA, van Heerden JA, Thompson GB, Schleck C, Ilstrup DM, Churchward M: Gastrointestinal carcinoid tumors: long-term prognosis for surgically treated patients. World J Surg 2000;24:1431-1436.

34 Pape UF, Berndt U, Muller-Nordhorn J, Bohmig M, Roll S, Koch M, Willich SN, Wiedenmann B: Prognostic factors of long-term outcome in gastroenteropancreatic neuroendocrine tumours. Endocr Relat Cancer 2008;15:1083-1097.

- 35 Edwards BK, Ward E, Kohler BA, Eheman C, Zauber AG, Anderson RN, Jemal A, Schymura MJ, Lansdorp-Vogelaar I, Seeff LC, van Ballegooijen M, Goede SL, Ries LA: Annual report to the nation on the status of cancer, 1975-2006, featuring colorectal cancer trends and impact of interventions (risk factors, screening, and treatment) to reduce future rates. Cancer 2010;116:544-573.

36 Gustafsson BI, Hauso O, Drozdov I, Kidd M Modlin IM: Carcinoid heart disease. Int J Cardiol 2008; 129:318-324.

37 Sobin L (ed): TNM Classification of Malignant Tumours. Oxford, Wiley-Blackwell, 2009.

38 Kulke MH: Neuroendocrine tumors: is there a standard treatment? Gastrointest Cancer Res 2008;2:152-153.

39 Hauso O, Gustafsson BI, Kidd M, Waldum HL, Drozdov I, Chan AK, Modlin IM: Neuroendocrine tumor epidemiology: contrasting Norway and North America. Cancer 2008;113:2655-2664.

40 Li AF, Hsu CY, Li A, Tai LC, Liang WY, Li WY, Tsay SH, Chen JY: A 35-year retrospective study of carcinoid tumors in Taiwan: differences in distribution with a high probability of associated second primary malignancies. Cancer 2008;112:274-283.

41 Reubi J, Waser B, Schaer J, Laissue J: Somatostatin receptor sst1-sst5 expression in normal and neoplastic human tissues using receptor autoradiography with subtypeselective ligands. Eur J Nucl Med 2001;28: 836-846.

42 Asnacios A, Courbon F, Rochaix P, Bauvin E, Cances-Lauwers V, Susini C, Schulz S, Boneu A, Guimbaud R, Buscail L: Indium-111-pentetreotide scintigraphy and somatostatin receptor subtype 2 expression: new prognostic factors for malignant well-differentiated endocrine tumors. J Clin Oncol 2008;26:963970

-43 Ambrosini V, Campana D, Bodei L, Nanni C, Castellucci P, Allegri V, Montini GC, Tomassetti P, Paganelli G, Fanti S: ${ }^{68} \mathrm{Ga}$-DOTANOC PET/CT clinical impact in patients with neuroendocrine tumors. J Nucl Med 2010;51:669-673.

44 Binderup T, Knigge U, Loft A, Federspiel B Kjaer A: ${ }^{18} \mathrm{~F}$-fluorodeoxyglucose positron emission tomography predicts survival of patients with neuroendocrine tumors. Clin Cancer Res 2010;16:978-985.
Nomogram to Assess Small-Intestinal Neuroendocrine Tumor Survival 